\title{
¿UNA FORMA DE COOPERACIÓN JUDICIAL NO RECLAMADA? SOBRE LA EXTENSIÓN DEL AMPARO A LA CARTA DE DERECHOS FUNDAMENTALES DE LA UE'
}

Uninvited remedy providers? On the repatriation of EU fundamental rights via constitutional adjudication

\author{
PEDRO CRUZ VILLALÓN \\ Universidad Autónoma de Madrid \\ p.cruz@uam.es
}

Cómo citar/Citation

Cruz Villalón, P. (2021).

¿Una forma de cooperación judicial no reclamada? Sobre la extensión del amparo a la Carta de Derechos Fundamentales de la UE. Anuario Iberoamericano de Justicia Constitucional, 25(1), 57-85. doi:https://doi.org/10.18042/cepc/aijc.25.03

\section{Resumen}

A lo largo de la última década los tribunales constitucionales de Austria y Alemania han efectuado sucesivamente un giro similar en su jurisprudencia anterior dirigido a incorporar el contenido sustantivo de la CDFUE a su jurisdicción de amparo. Partiendo de las deficiencias de la Carta en lo relativo a la garantía jurisdiccional de los derechos en ella reconocidos, el artículo analiza y compara esta jurisprudencia

1 El presente texto tiene origen en mi intervención en el «XX. Walter Hallstein Kolloquium» celebrado el pasado 4 de marzo en la Universidad de Francfort/Meno con el título «Kooperativer Grundrechtsschutz in der Europäischen Union. Aktuelle Entwicklungen im Lichte der neuen Rechtsprechung des Bundesverfassungsgerichts und des EuGH». Agradezco muy en especial al Prof. Dr. Rainer Hofmann el haberme proporcionado las claves imprescindibles de la jurisprudencia del Tribunal Constitucional Federal aquí objeto de evaluación y comentario. 
desde la perspectiva de las referidas deficiencias a la vez que plantea la cuestión de si el Tribunal Constitucional de España debiera seguir el ejemplo de los referidos tribunales.

\section{Palabras clave}

Carta de Derechos Fundamentales de la Unión Europea; recurso de amparo constitucional; tribunales constitucionales.

\section{Abstract}

The past decade has seen the Austriand the German Constitutional Courts successively engaging in a similar U-turn in their former case-law in order to adjudicate on individual complaints (Verfassungsbeschwerde) vindicating fundamental rights enshrined in the EU Charter. The article analyzes the resulting constitutional case law focusing on its implications on the normativity of the Charter and inquires on the likelihood of corresponding developments in other Member States with similar constitutional remedies as is prominently the case of the Spanish amparo.

\section{Keywords}

Charter of Fundamental Rights of the European Union; Individual Complaints; Constitutional Courts. 


\section{SUMARIO}

I. LA ORFANDAD ORIGINARIA DE LOS DERECHOS DE LA CARTA. II. LA PULSIÓN IUSPOSITIVISTA: VIENA, 2012. III. LA PULSIÓN IUSNATURALISTA: KARLSRUHE, 2019. IV. LA PULSIÓN QUIETISTA: MADRID, 2021. V. CONCLUSIONES. BIBLIOGRAFIA.

«Las demandas de amparo deben ser estimadas. Las sentencias impugnadas vulneran el derecho fundamental de los demandantes ex artículo 4 CDFUE».

Sentencia del Tribunal Constitucional Federal alemán (Sala Segunda) de 1 de diciembre de 2020, punto $33^{2}$.

Las breves líneas de la cita jurisprudencial que antecede, más que improbables en boca del Tribunal Constitucional de España, son expresivas de una evolución no menor en la justicia constitucional a nivel europeo ${ }^{3}$. A lo largo de la pasada década y por la vía de llamativos cambios en su jurisprudencia, dos tribunales constitucionales han coincidido en la incorporación de los contenidos sustantivos de la Carta de Derechos Fundamentales de la Unión Europea (en adelante, CDF) al ámbito de tutela de sus respectivos recursos constitucionales de amparo (Verfassungsbeschwerde) ${ }^{4}$. Es el caso de los dos tribunales que, junto con el español, forman la tríada más reconocida en lo que se refiere a la jurisdicción constitucional de amparo en el espacio europeo: el Verfassungsgerichtshof austriaco y el Bundesverfassungsgericht alemán. En tanto el primero hizo labor de pionero en esta aventura, cuando la CDF apenas llevaba en vigor dos años ${ }^{5}$, el segundo ha seguido mucho más recientemente

2 Con reflejo inmediato en el correspondiente punto del fallo.

3 De hecho, los comentaristas no se han quedado cortos en los calificativos: «nueva era» (Hofmann y Heger, 2021: 1), «espectacular» (Pöschl, 2012: 587), «instant classic» (Orator, 2015: 1429).

4 Desde el propio título de este trabajo me permito la licencia, por economía del lenguaje, de referirme frecuentemente con el término español de «amparo» al remedio judicial basado en la demanda individual de protección de los derechos fundamentales que tienen encomendado algunos tribunales constitucionales, en definitiva, la Verfassungsbeschwerde.

5 Sentencia de 14 de marzo de 2012 (U 466/11-18 y U 1836/11-13). Versión en inglés accesible en https://bit.ly/2PWjdXl. 
sus pasos ${ }^{6}$, por más que cada uno con perfiles propios. Y si durante la mayor parte de este periodo la iniciativa del tribunal vienés pudo ser vista desde fuera como una originalidad nacional, la incorporación de la jurisdicción constitucional alemana a esta línea jurisprudencial invita a analizarla en términos de posible modelo, acaso a seguir por otras jurisdicciones constitucionales y señaladamente la española ${ }^{7}$.

Estas páginas se proponen analizar esta evolución jurisprudencial en paralelo en lo que tiene de avances en la construcción de un sistema de remedios judiciales de garantía de los derechos de la Carta. El fenómeno interesa en su consecuencia europea, es decir, en la medida en que esta modificación de la jurisdicción constitucional de derechos fundamentales se traduce, por decirlo con cautela, en la promesa de un salto cualitativo en la garantía de los derechos de la CDF. No se trata, por tanto, de abordar el conjunto de expedientes, así como las diversas modalidades adoptadas por las jurisdicciones de los Estados miembros en orden a asumir a nivel interno la normatividad de la CDF desde 2009, lo que excedería de las dimensiones de un escrito de estas características ${ }^{8}$. El objetivo es el de centrarse en el fenómeno mucho más concreto consistente,

6 Mediante sentencia de su Sala Primera de 6 de noviembre de 2019 (1 BvR 276/17) conocida allí como Derecho al olvido II (Recht auf Vergessen II): Traducción completa al inglés y al francés accesible respectivamente en https://bit.ly/3etuBDM y en https:// bit.ly/2RzgDqV. «Derecho al olvido II» por cuanto precedida de la sentencia de la misma fecha "Sentencia al olvido I» (1 BvR 16/13), a lo que más adelante se hará referencia (traducción al inglés accesible en https://bit.ly/3b7oh2o y al francés en https:// bit.ly/3vM6SnY. Jurisprudencia confirmada mediante sentencia de la Sala Segunda el 1 de diciembre de 2020 (2 BvR 1845/18 y 2 BvR 2100/18), conocida allí como «Euroorden III» (Europäischer Haftbefehl III), resumen accesible en inglés, https://bit. ly/3nTBcuf.

7 Es de tener en cuenta que, en concreto en el caso de Alemania, el cambio jurisprudencial venía precedido de una importante reflexión doctrinal, de la que conviene destacar la de Claudio Franzius ya en 2015 (pp. 148-152).

8 La materia se ha vuelto ya casi inabordable. Una concisa presentación de estas diversas modalidades en Wendel, 2020: 1387-1394. Cfr., para el Tribunal Constitucional español, Arzoz, 2015. Es de notar ya cómo Derecho al olvido II (punto 50) hace un esfuerzo por situar su cambio jurisprudencial en la compañía de iniciativas semejantes de otros tribunales. En realidad, el único supuesto comparable, con carácter de precue$l a$, es el austriaco. Con esto no se pretende restar toda la importancia que tienen algunos desarrollos muy recientes: valga citar, por ceñirse a un solo ejemplo, por el lado del TJUE, Consob, Sentencia de 2 de febrero de 2021 (C-481/19, ECLI:EU:C:2021:84) en el actual "periodo de reajuste» en el marco del diálogo entre tribunales europeos (Ahumada, 2020: 249). 
vale repetir, en una jurisdicción constitucional que amplía a los derechos de la Carta el remedio judicial nacional de garantía de los propios derechos fundamentales puesto a disposición de los particulares. Por último, el contraste entre la jurisprudencia emanada por cada uno de estos dos tribunales ofrece el interés adicional de poner de manifiesto la pervivencia de dos tradiciones jurídicas nacionales claramente diferenciadas, lo que llamaré la pulsión iuspositivista en un caso y iusnaturalista en el otro.

Punto de partida obligado será el contexto en el que se sitúan los derechos de la Carta desde la perspectiva de su tutela jurisdiccional (1). Sucesivamente procederá describir los rasgos tanto de la iniciativa austriaca (2) como los de la alemana (3) en los aspectos que más importan, dando paso así a la reflexión anunciada sobre las perspectivas que con lo anterior acaso puedan abrirse para la jurisdicción constitucional en España (4).

\section{LA ORFANDAD ORIGINARIA DE LOS DERECHOS DE LA CARTA}

La CDF, peculiar por tantos motivos, lo es sin duda también por la despreocupación de sus autores en lo relativo a un extremo vital en toda declaración de este tipo, la garantía jurisdiccional de los derechos en ella proclamados. La más atenta lectura de sus enunciados no alcanza a descubrir rastro alguno de inquietud por dicha tutela o garantía. Mejor dicho: el único pasaje de la Carta en el que se aborda esta problemática tiene por objeto precisamente rebajar las expectativas de garantía en un determinado ámbito. Es así como en el apdo. 5 de su art. 52 se declara, en una transcripción libre, que las disposiciones de la CDF que contienen principios solo pueden alegarse ante un órgano jurisdiccional en lo que se refiere a la interpretación y legalidad de los eventuales actos legislativos y ejecutivos adoptados por las instituciones de la Unión, o por los Estados miembros cuando apliquen el derecho de la Unión. Debe ahora dejarse de lado la cuestión de cuáles sean las disposiciones así concretamente aludidas. Baste ahora señalar que dichas disposiciones no pueden ser invocadas directamente, en ausencia de actos dirigidos a su desarrollo o aplicación?.

Cierto es que una interpretación a contrario del referido apdo. 5 podría llevar a entender que, de modo implícito, el resto de las disposiciones de la Carta sí serían directamente alegables ante los tribunales. En seguida lo veremos. Lo que importa ahora es que la CDF no contiene ninguna previsión

9 Me remito, por lo que todavía puedan valer, a las conclusiones en el asunto Association de médiation sociale (C-176/12, ECLI:EU:C:2013:491), puntos 57-72. 
específica, en positivo, respecto de la garantía de sus contenidos. Esta ausencia es ciertamente explicable en términos históricos: la CDF nació, como es sabido, y así se mantuvo durante una década, carente de valor jurídico, de tal modo que difícilmente podía contener previsión alguna relativa a su garantía. Pero precisamente ese argumento histórico deja de ser tal desde el momento en que, con el Tratado de Lisboa, la Carta pasa a tener valor jurídico y en concreto valor de derecho primario. En definitiva, una vía de recurso singularizada destinada a la protección de los derechos de la Carta no aparece ni en la propia CDF ni en el resto del derecho primario. $\mathrm{Al}$ igual que tampoco exige el derecho de la Unión que el ordenamiento de los Estados miembros prevea tal cosa para los supuestos de aplicación de la Carta en sus respectivas actuaciones públicas. Cabría incluso preguntarse si desde la Unión y en concreto desde el TJUE no se estaría viendo como una oficiosidad la evolución que nos ocupa.

De lo anterior resulta que la protección que corresponde a los derechos fundamentales de la CDF debe ser remitida a la ordinaria que resulta de su art. 47 («Derecho a la tutela judicial efectiva y a un juez imparcial»): de manera general, pues, toda persona tiene derecho a la tutela judicial de los derechos y libertades que el derecho de la Unión le reconoce, de los que sería absurdo excluir a los derechos y libertades de la CDF.

La cuestión, llegados a este punto, es la de quién sea el obligado o destinatario de la garantía judicial de tales derechos subjetivos, fundamentales o no, dicho en otros términos, a quién corresponde poner en pie esa tutela judicial efectiva. La respuesta es simple: al Tribunal de Justicia de la Unión Europea en primerísimo lugar y, a partir de ahí, a los jueces y tribunales de los Estados miembros. Más concretamente, al Tribunal de Justicia respecto de los actos de las instituciones y órganos de la Unión que tengan la condición de inmediatamente aplicables, a los jueces y tribunales de los Estados miembros, de manera interpuesta, cada vez que ese derecho de la Unión requiere la intermediación de un acto nacional.

Esto implica que, por lo que hace al primero de los supuestos, la tutela de los derechos de la Carta no es de mejor condición que la ordinaria de cualesquiera derechos e intereses legítimos. Lo cual tiene su importancia teniendo en cuenta que esta tutela ordinaria viene padeciendo desde los primeros años de la instalación de la jurisdicción de la hoy Unión Europea del régimen draconiano derivado de la doctrina Plaumann respecto de lo que se llaman los justiciables no privilegiados, vale decir, los particulares ${ }^{10}$. A este respecto

10 Vale recordar cómo Plaumann \& Co (Sentencia de 15 de julio de 1963, C-25/62) continúa a la fecha de hoy declarando «que quienes no sean destinatarios de una Decisión sólo pueden alegar que ésta les afecta individualmente cuando dicha Decisión les 
resulta inevitable añadir que las «Explicaciones sobre la Carta» en lo que se refiere a este art. 47, apuntan inequívocamente a un no cuestionamiento de esta tozuda jurisprudencia ${ }^{11}$. En todo caso, y por más que forme parte importante del contexto, no es el objeto de estas páginas tratar de esta vertiente de la tutela de los derechos de la Carta, la que corresponde inmediatamente al TJUE.

La cuestión que nos ocupa, vale reiterar, es la de los supuestos en los que la tutela judicial de los derechos de la Carta es responsabilidad de los Estados miembros, siendo aquí donde se sitúa la cuestión, verdaderamente crucial, de cuáles sean los actos de los Estados miembros que, en los términos del art. 51.1 CDF aplican el derecho de la Unión con la consecuencia de que sus contenidos sustantivos desplazan a los derechos fundamentales constitucionalmente garantizados en los Estados miembros. No hace falta decir que la cuestión se encuentra hoy respondida en los conocidos términos de principio que se contienen en la sentencia Akerberg ${ }^{12}$, sucesivamente objeto de matizaciones dispersas ${ }^{13}$.

Dicho muy simplemente, la cuestión hoy día es que los actos de poder público en los Estados miembros e incluso, en su caso, los actos de libre dispo-

atañe debido a ciertas cualidades que les son propias o a una situación de hecho que les caracteriza en relación con cualesquiera otras personas y, por ello, les individualiza de una manera análoga a la del destinatario». Una propuesta todavía digna de ser leída, Kottmann, 2010: 547-566.

11 En los siguientes términos: «[...] La inclusión de esta jurisprudencia en la Carta no tenía por objeto modificar el sistema de control jurisdiccional establecido en los Tratados ni, en particular, las normas relativas a la admisibilidad de los recursos interpuestos directamente ante el Tribunal de Justicia de la Unión Europea. La Convención Europea ha examinado el sistema de control jurisdiccional de la Unión, incluidas las normas relativas a la admisibilidad y ha confirmado dicho sistema, si bien se han modificado determinados aspectos, como establecen los artículos 251 a 281 del Tratado de Funcionamiento de la Unión Europea y, en particular, el párrafo cuarto del artículo 263». Como es bien conocido, de poco ha servido la modificación aludida contenida en el citado párrafo cuarto del art. 263 TFUE. Valga la remisión a la suerte de los asuntos Inuit (C-583/11P, ECLI:EU:C:2013:625), Telefónica (C-274/12 P, ECLI:EU:C:2013:852) y T\&L Sugars Ltd. (C-456/13 P, ECLI:EU:C: 2015:284), conclusiones de 14 de octubre de 2014 (ECLI:EU:C:2014:2283).

12 Aklagaren contra Hans Akerberg Fransson, Sentencia de 26 de febrero de 2013 (C617/10, ECLI:EU:C:2013:105).

13 Hasta llegar a TSN (C-609/17 y C-610/17, ECLI:EU:C:2019:981), por poner un claro ejemplo de relativización de las rotundas frases de Akerberg (punto 21). Una recopilación a la altura de 2017 facilitada por el TJUE, accesible en https://bit.ly/2R2eROT. 
sición de los particulares en dichos Estados miembros se encuentran sometidos al respeto de dos series, en buena medida paralelas, de derechos fundamentales, la que se deriva del ordenamiento constitucional respectivo y la común que se deriva de la CDF. Y que los órganos jurisdiccionales nacionales están llamados a operar con ambas series de derechos fundamentales, muchas veces coincidentes en su enunciado y contenido. Todo ello dejando de lado por el momento la alargada sombra del CEDH.

A ello se suma la circunstancia de que, en algunos Estados miembros, una jurisdicción separada tiene atribuida de manera singularizada la garantía de la Constitución nacional y en particular de los derechos fundamentales en ella declarados como una parte esencial de su razón de ser. En algunos casos, los que aquí nos ocupan, esta garantía constitucional de los derechos fundamentales toma la forma de un remedio singular a disposición de los particulares, el recurso de amparo o Verfassungsbeschwerde. Con ello ya se está diciendo que, como cuestión de partida, los derechos fundamentales llamados a ser garantizados por esta jurisdicción separada son los que integran una de las referidas series, la que figura en la respectiva Constitución nacional. En esto, la doctrina constitucional, en los Estados miembros considerados, ha sido estable y pacífica $^{14}$ : la jurisdicción constitucional no tiene atribuida la tutela de la otra serie de derechos, la que ahora se contiene en la CDFUE. Basten estas someras notas para pasar a describir el modo cómo los dos tribunales nacionales reiteradamente citados han modificado esta jurisprudencia.

\section{LA PULSIÓN IUSPOSITIVISTA: VIENA, 2012}

La primera jurisdicción constitucional de amparo en reaccionar frente al potencial perturbador que la entrada en vigor de la Carta como derecho primario podría suponer para su respectiva función jurisdiccional es, como se ha indicado, el Verfassungsgerichtshof vienés. Lo hace en un momento en el que las grandes incógnitas interpretativas que, en particular, el título VII de la Carta supone, apenas están siendo abordadas por el TJUE: Åkerberg todavía tardará un año en llegar ${ }^{15}$.

14 Así desde el primer momento en el caso de las jurisdicciones nacionales correspondientes a Estados incorporados a la hoy Unión Europea en un posterior momento (España y Austria). Como es conocido, el tribunal alemán debió hacer su propia evolución hasta esencialmente Solange II, en 1986 (BVerfGE 73, 339, de 22 de octubre).

15 Toda la complejidad del estado de la cuestión a la altura de 2012, en Iglesias Sánchez (2012: 1656-1612). 
En el origen de los dos amparos acumulados que dan lugar a la en seguida llamada, por antonomasia, "Sentencia de la Charta» (Charta-Erkenntnis), se encuentra la queja de personas de nacionalidad china a las que el órgano jurisdiccional singular al efecto previsto (Asylgerichtshof) deniega el asilo sin otorgar la oportunidad de una comparecencia oral a la que los demandantes consideran tener derecho. Se da el caso de que esta vía de recurso ha sido oportunamente incorporada a la Constitución (art. 144a) en un contexto nacional en el que el amparo constitucional, sin necesidad de entrar en sus detalles, dista de ser el remedio universal frente a resoluciones judiciales tal como aparece en Alemania ${ }^{16}$.

La singularidad de los casos en cuestión estriba en que estos demandantes de amparo no invocan el derecho fundamental a la tutela judicial constitucionalmente reconocido, que por lo que se recordará no es otro en Austria que el CEDH. El derecho invocado será el más ampliamente regulado en el art. $47 \mathrm{CDF}$. Ahora bien, apenas hace falta recordar que, con arreglo a la doctrina constante del Verfassungsgerichtshof, que este recordará puntualmente, este no es juez del derecho de la Unión ${ }^{17}$. Son los diversos órdenes jurisdiccionales ordinarios los llamados a aplicarlo. En suma, una admisión de estas demandas de amparo no resultará posible sin proceder a un notable overruling. Es lo que hará, como se viene indicando, en la reiterada sentencia de 14 de marzo de 2012. Su modo de argumentar este cambio nos interesará particularmente.

El tribunal vienés hará gala en esta sentencia de una pulsión iuspositivista, casi se diría que instalada en su ADN. Podría considerarse también que para argumentar este cambio el tribunal no va a necesitar echar mano de principios generales, que el derecho escrito le basta y sobra. En este sentido, buscará en primer lugar argumentar por qué su cambio de jurisprudencia debe tener lugar en este momento temporal, el de la positivación de los derechos fundamentales de la Unión. El tribunal explicará esto de dos maneras, una más que formalista, otra de bastante más calado. En primer lugar, el tribunal advierte que, con arreglo a su jurisprudencia, lo que se consideraba un canon de control situado fuera de su jurisdicción eran «los Tratados», es decir, los tratados constitutivos de la Unión, ahora el TUE y el TFUE. Ahora bien, cuando el art. 6.1 TUE declara que, en la versión alemana, «la CDF y los Tratados tienen el mismo valor jurídico» estaría poniendo de manifiesto que una cosa son los tratados y otra la CDF, para la que, por tanto, no tendría que

16 Sobre la relativa "paridad» de los tres altos tribunales austriacos, Constitucional, Supremo y Administrativo (Verfassungsgerichtshof, Oberster Gerichtshof y Verwaltungsgerichtshof) véanse Grabenwarter (2016: 454-457), Orator (2015: 1431-1432).

17 Puntos 19-24. 
valer necesariamente la anterior jurisprudencia ${ }^{18}$. Dígase simplemente que no es el mejor razonamiento salido de la boca del tribunal vienés.

El argumento de verdadero peso, el que de forma más patente revela la pulsión iuspositivista del Verfassungsgerichtshof, es el que se centra en el cambio de paradigma que la entrada en vigor de la CDF implica: en concreto, el que supone el paso de unos derechos fundamentales europeos carentes de específica plasmación en el ordenamiento de la Unión, considerados, sin embargo, parte de los principios generales de ese derecho, a una declaración positivizada de los mismos como la que lleva a cabo la CDF. Sencillamente, "no son comparables»: "El valor de un catálogo detallado de derechos y deberes como el que contiene la Carta de Derechos Fundamentales no es, sin embargo, comparable al que resulta de la deducción de posiciones jurídicas a partir de principios generales del Derecho ${ }^{19}$.

Resulta imposible, hasta donde se me alcanza, encontrar una declaración similar en boca de otro tribunal constitucional acerca de la trascendencia de este cambio de paradigma: el del paso de un sistema de derechos obtenido mediante operaciones deductivas a un sistema sencillamente constitucional de derechos formulados y declarados. No es en absoluto casual que sea en Viena donde se pergeña una formulación como esta. La Carta en lo que tiene de positivación de los derechos tendría un potencial propio que el juez constitucional no puede ignorar. Esta pulsión iuspositivista encuentra su prolongación en una serie de argumentos adicionales, que poco tienen de principialistas.

Con arreglo al derecho de la Unión, más allá de la autonomía procesal de los Estados miembros, estos tienen vedado que sus jueces y tribunales otorguen al derecho de la Unión una efectividad inferior a la que otorgan a su propio derecho (principio de equivalencia): es la jurisprudencia que arranca de Rewe, oportunamente citada por el tribunal austriaco ${ }^{20}$. De donde este extrae la conclusión de que lo mismo que los derechos fundamentales nacionales, los de la Carta deben ser también objeto de protección por medio del amparo ante la jurisdicción constitucional. Se trata esta de una conclusión

18 5. «Esta jurisprudencia relativa al Derecho de la Unión Europea emitida con anterioridad a la entrada en vigor del Tratado de Lisboa no puede ser trasladada a la Carta de Derechos Fundamentales. Ella constituye en el conjunto del Derecho de la Unión un ámbito claramente delimitado respecto de los "Tratados" (vid. art. 6.1 TUE: "la Carta de los derechos fundamentales y los Tratados"), respecto de la cual y con base en el ordenamiento constitucional interno su tratamiento ha de ser diferente)» (traducción nuestra).

19 Punto 38

20 Puntos 26-28. Rewe-Zentral AG, Sentencia de 20 de febrero de 1979 (C-33/76, ECLI:EU:C:1979:42) (Prechal, 2011: 31-50). 
atípica (Orator, 2015: 1439), con la que el tribunal austriaco busca que las cuestiones de interpretación de la CDF le lleguen en la misma intensidad que le llega la interpretación y garantía de los derechos constitucionalmente garantizados.

Por fin, habría un argumento estructural a favor del cambio jurisprudencial, que el tribunal vienés extrae del propio modelo de control concentrado de constitucionalidad, al que, como la doctrina ha señalado, pretende dar un alcance muy superior al real ${ }^{21}$. Cierto es que las demandas de amparo frente a actos de los poderes públicos van directamente al Verfassungsgerichtshof, sin pasar por el Verwaltungsgerichtshof. Como también es cierto que solo él controla la constitucionalidad de las normas generales con efecto ordinario de derogación. Pero, en particular, la jurisdicción civil y penal hasta llegar al Oberster Gerichshof siempre ha descartado la aplicación incidental de las leyes inconstitucionales, derechos fundamentales incluidos ${ }^{22}$.

Hasta aquí los argumentos esenciales que deben apuntalar el cambio de doctrina. Por lo que hace a la organización de la nueva competencia que el tribunal austriaco se atribuye, debe señalarse en primer lugar un extremo concerniente al contenido sustantivo de la CDF que pasará a disfrutar de la tutela por vía de amparo. A lo largo de la CDF y en particular en el caso de los «principios» en el sentido del título VII, se advierte la presencia de preceptos que muestran "una estructura normativa totalmente diferente» que hace imposible que funcionen como parámetro adecuado de control. La «Sentencia de la Carta» es poco más precisa a este respecto, pero, en definitiva, detrás de este razonamiento late la idea de una reducción, a efectos del amparo, de los contenidos sustantivos de la CDF, a aquellos que corresponden a los derechos constitucionalmente garantizados y en definitiva al $\mathrm{CEDH}^{23}$.

En segundo lugar, y a diferencia de lo que será el caso alemán, el cambio jurisprudencial va más allá de la demanda individual de amparo (Verfassungsbeschwerde). El tribunal pretende ser consultado incidentalmente también respecto de la interpretación de los referidos contenidos sustantivos de la Carta, con las consecuencias que en seguida se verán. Por fin, crucial en toda esta materia son las consecuencias sobre el deber de la jurisdicción constitucional de asumir la posición de órgano jurisdiccional en el sentido del art. 267 TFUE, en concreto, de órgano de última instancia nacional obligado a dirigirse al TJUE en petición de interpretación del derecho de la Unión salvo

21 Puntos 33-35. Pöschl, 2012: 595: «Das ist neu und das ist falsch».

22 Ibid., nota 21.

23 Punto 34. 
en caso de acto claro o aclarado en el sentido de CILFIT ${ }^{24}$. En efecto, y este es un extremo que compartirá con la nueva doctrina alemana, la decisión del tribunal austriaco de incorporar parte de los contenidos sustantivos de la CDF al ámbito del amparo acarrea la consecuencia de convertirse, de forma mucho más ordinaria, en órgano jurisdiccional de última instancia sometido al deber de plantear una cuestión prejudicial de interpretación al TJUE sobre el derecho fundamental eventualmente a amparar, salvo en los conocidos supuestos de acto claro o aclarado. El Verfassungsgerichtshof, como años más tarde su homólogo alemán, hará una proclamación, más o menos sincera, de acatamiento de este mandato ${ }^{25}$.

Ahora bien, en Austria se da adicionalmente la conocida y singular circunstancia de que el $\mathrm{CEDH}$ tiene rango constitucional. Es más, el Convenio funciona prácticamente como «la parte dogmática» de la Constitución austriaca teniendo en cuenta que la Constitución de 1920 simplemente incorporó los viejos derechos fundamentales de la llamada «Constitución de Diciembre», de 1867, con la calificación, desde entonces, de nuevo bien neutra de "derechos constitucionalmente garantizados» ${ }^{26}$. Si esto a su vez se combina con la circunstancia de que la CDF contiene el conocido mandato de equiparación interpretativa de ambas series de derechos en el apartado tercero de su art. 52, el tribunal extrae una consecuencia adicional, con la consiguiente repercusión en su deber de formular cuestiones prejudiciales. En efecto, con base en la circunstancia recién indicada, el tribunal vienés realiza una pirueta, de nuevo discutida por la doctrina ${ }^{27}$ que neutraliza en buena medida el deber de acudir incidentalmente a Luxemburgo: puesto que con enorme frecuencia los derechos de la CDF coincidirán con los del CEDH, que casualmente son también los derechos constitucionales austriacos, la cuestión de interpretación de la CDF no será determinante para el fallo porque vendrá resuelta con base en la otra serie de derechos, los del CEDH, que son al mismo tiempo los austriacos.

Por lo que hace al destino de esta jurisprudencia conviene comenzar señalando que esta fue sometida casi en seguida al control por parte del TJUE por la vía de una cuestión prejudicial del Tribunal Supremo (Oberster Gerichtshof) en un extremo concreto, bastante semejante al ya resuelto por Luxemburgo en

24 Sentencia de 6 de octubre de 1982, ECLI:EU:C:1982:335. Sugiriendo un replanteamiento, Sarmiento (2010: 199-200).

25 De momento, todos los supuestos aquí contemplados se han resuelto sin planteamiento de cuestión prejudicial.

26 Grabenwarter (2016: 420-422).

27 Pöschl (2012: 598). 
el asunto Melki y Abdeli $^{28}$. En realidad, sabemos cómo el tribunal vienés ya había expresado su acatamiento expreso de la referida doctrina. Esta cuestión prejudicial hubiera dado al TJUE la ocasión, finalmente perdida, de hacer una valoración de la oferta proveniente de una jurisdicción constitucional de amparo de reforzar la garantía jurisdiccional de la CDF por medio de un instrumento procesal de tanta intensidad como es la Verfassungsbeschwerde. La sentencia se limita a reiterar con matizaciones ${ }^{29}$ la doctrina contenida en Melki y Abdeli, dejando pasar la oportunidad de saludar, al menos en su intención, la jurisprudencia nacional ${ }^{30}$.

La «sentencia de la Carta», como ya se ha venido avanzando, no fue recibida particularmente bien por la doctrina, entre otras cosas por sus cuestionables consecuencias prácticas. Sí se apuntó (Pöschl, 2012: 608) que las cosas podrían ser distintas si saliera adelante, como así ocurrión ${ }^{31}$, el proyecto de incorporar a la Constitución una vía de recurso frente a normas con rango de ley a favor de quienes en el curso de un proceso civil o penal hubieran sido objeto de aplicación de tales normas (Gesetzesbeschwerde). En tal caso, la incorporación de la CDF como parámetro en tal modalidad de proceso adquiriría nuevo sentido. No parece que, de manera general, esta nueva vía de recurso y en general el cambio jurisprudencial de 2012 haya tenido las consecuencias que cabría derivar de su importancia intrínseca en términos de principio. Digital RightsIreland y Seidlinger parece ser la última ocasión en la que el tribunal austriaco formuló una cuestión prejudicial ${ }^{32}$. Rauchegger (2020: 270) hace un expresivo balance de los años transcurridos: en la inmensa mayoría de los casos el tribunal vienés se contenta con indicar

28 Aziz Melki y Selim Abdeli, Sentencia de 22 de junio de 2010 (C-189/10 y 189/10, ECLI:EU:C:2010:363).

29 De Visser, 2015:1320.

30 Varios comentaristas, entre ellos De Visser (2015:1334 y nota 89, con remisión a nota 48), se hacen eco de una carta del presidente del TJUE, Vassilios Skouris, dirigida al Verfassungsgerichtshof, aparentemente con ocasión de esta jurisprudencia, en la que se vierte el parecer de que la incorporación del canon de la CDF al amparo constitucional contribuiría a hacer de la misma parte integrante del patrimonio común europeo.

31 Con efectos de 1 de enero de 2015. Pöschl (2012: nota 37) señala la coincidencia de que la sentencia se hiciera pública dos días después de que el Verfassungsausschu $\beta$ diera luz verde a esta reforma constitucional.

32 Con arreglo a la página del Verfassungsgerichshof: Sentencia de 8 de abril de 2014 (C-293/12, ECLI:EU:C:2013:238), https://bit.ly/3urkSDi. Cabe señalar que fue el Oberster Gerichtshof quien planteó la cuestión prejudicial relativa al disfrute discriminatorio del festivo del Viernes Santo: Cresco Investigation $G m b H$, Sentencia de 22 de enero de 2019 (C-193/17, ECLI:EU:C:2019:43). 
de forma bastante lacónica que la CDF no otorga una protección superior al propio régimen de derechos.

\section{LA PULSIÓN IUSNATURALISTA: KARLSRUHE, 2019}

Hay que esperar a 2019, cuando la CDF cumple diez años de vigencia, para encontrar una jurisprudencia constitucional nacional equivalente a la austriaca. El 6 de noviembre de ese año, la Sala Primera del Tribunal Constitucional Federal alemán pronuncia dos sentencias coordinadas, conocidas como Derecho al olvido I y Derecho al Olvido II ${ }^{33}$, la segunda de las cuales proclama el acogimiento de los derechos de la Carta a la tutela dispensada por su propio recurso de amparo (Verfassungsbeschwerde). De este modo el tribunal alemán se aparta de lo que venía siendo su jurisprudencia desde Solange II fundada en lo que terminó llamándose la «teoría de la separación» (Trennungstheorie): dicho sencillamente, cada tribunal, el europeo y el nacional, es responsable exclusivo de la efectividad y garantía de su respectivo ordenamiento, derechos fundamentales incluidos, todo ello con reflejo inmediato en la jurisdicción de amparo (Franzius, 2015: 148). Conviene de nuevo comenzar por una serie de observaciones relativas al contexto de esta jurisprudencia.

En primer lugar, y a diferencia de lo que ocurrió en Viena, a Karlsruhe no llega ningún demandante de amparo reivindicando un derecho de la Carta: será el propio tribunal quien transforme las demandas de amparo basadas en un precepto de la Grundgeset $z^{34}$ en una demanda de un derecho fundamental

33 Cit. nota 3.

34 Esta vez el derecho fundamental invocado en ambas demandas de amparo es esencialmente el derecho a la protección de datos, sub specie autodeterminación informativa (art. 2.1 en conexión con el art. 1.1 Grundgesetz). En Derecho al olvido I se reclama, y en definitiva obtiene, el borrado de una referencia personal del demandante, relativa a su condena penal por asesinato en 1982, disponible en una base de datos del semanario Der Spiegel. En Derecho al olvido II la demandante de amparo reaccionaba de manera similar, pero sin el mismo éxito, frente al libre acceso a su nombre vía Google en el contexto de una determinada emisión televisiva («Panorama», emitida en 2010, Norddeutsche Rundfunk), donde quedaba en entredicho su actuación en tanto que empleadora. A diferencia de lo que era el caso austriaco, aquí estamos ante supuestos de eficacia horizontal de la CDF, que el tribunal alemán acepta sin reservas, y más en concreto de ponderación de derechos, en este caso con los derechos a la libertad de expresión en el primer caso, y el derecho a la libertad de empresa, en el segundo. 
europeo $^{35}$. Ello requerirá argumentar específicamente esta circunstancia. A estos efectos puede decirse ya que su teoría del «equivalente funcional» entre ambas series de derechos le facilitará la tarea.

Más interés tiene la circunstancia de que, a diferencia de lo que fue el caso austriaco y como se acaba de señalar, Derecho al olvido II forme pareja con Derecho al olvido $I$. La funcionalidad pretendida de este emparejamiento no se diferencia mucho de la revelada por el TJUE uniendo a Åkerberg y Melloni ${ }^{36}$ el ya lejano 26 de febrero de 2013. También aquí ambas sentencias se apoyan mutuamente. Es más, toda la construcción de la pareja alemana resulta de una especie de summa divisio, construida a partir de lo que podríamos calificar como la «ecuación 29/60», en referencia al punto 29 de Akerberg repetido en el 60 de Melloni. Derecho al Olvido I, del que no vamos a hablar más aquí, es en buena medida una reinterpretación a nivel nacional de la tolerada resiliencia de los derechos fundamentales nacionales en los supuestos en los que una materia solo es regulada de manera imperfecta por el derecho de la Unión. En los términos sabidos, cuando hay regulación por parte de la Unión, pero no de forma acabada, pueden regir los derechos fundamentales estatales siempre que este control no dañe a la primacía, unidad y efectividad del derecho de la Unión ${ }^{37}$. Es lo que proclama la referida «ecuación 29/60». Para el tribunal alemán este es esencialmente el momento del pluralismo europeo en materia de derechos fundamentales.

A contrario sensu, en las situaciones en las que, por defecto, el derecho de la Unión regula acabadamente una materia, la consecuencia ineluctable es que los derechos de la CDF desplazan enteramente a los derechos fundamentales nacionales concernidos. Es aquí donde Karlsruhe, por medio de Derecho al olvido II engancha el cambio jurisprudencial que nos viene ocupando: la regulación acabada de una materia por el derecho de la Unión tiene ciertamente la consecuencia de desplazar a los derechos fundamentales de la Grundgesetz, pero, a diferencia de lo que se venía entendiendo hasta ahora, y esta es la gran proclamación, no tiene la consecuencia de desplazar la jurisdicción del Tribunal Constitucional Federal ${ }^{38}$. Este será el momento de la uniformidad

35 Llama la atención que baste al efecto recordar la condición del tribunal como «dueño del proceso» (Hofmann y Heger, 2021: 3: «Herr des Verfahrens»).

36 Stefano Melloni (C-399/11, ECLI:EU:C:2013:107).

37 Reserva sobre la que Derecho al olvido I pasa como sobre ascuas (punto 48).

38 Ahora bien, lo hasta ahora dicho supone ya, dicho incidentalmente, que toda la construcción contenida en esta pareja jurisprudencial se apoya en una categoría que dista de ser unívoca, la del carácter acabado o inacabado, según los casos, de la regulación de una materia por el Derecho de la Unión: en su terminología, «Derecho (nacional) completamente determinado» frente a «Derecho (nacional) no completamente de- 
en el espacio europeo de los derechos, pero será el tribunal nacional quien se ocupe de su garantía.

Último elemento a destacar en relación con el contexto es que, de momento y durante un año cumplido, Derecho al olvido II es una jurisprudencia de una de las dos Salas, y precisamente de la primera, que no es la que lleva el peso de los derechos fundamentales con arreglo a la distribución de responsabilidades en el interior de este tribunal bicéfalo ${ }^{39}$. De ahí la importancia otorgada e incluso el alivio con el que fue recibida la Sentencia del pasado 1 de diciembre de la Sala Segunda Euroorden III ${ }^{40}$. La sentencia confirma sin reservas, aun sin necesidad de ser reproducción literal, la doctrina de su Sala gemela. Introduce pluralidad en el ámbito de esta nueva competencia al ocuparse de un derecho diferente, la prohibición de tratos inhumanos o degradantes del art. 4 CDF. Y tiene la importancia, frente a Derecho al olvido II, de declarar con toda rotundidad y naturalidad que se otorga el amparo por violación de un derecho de la Carta: es la cita que abre estas páginas.

Pasando ya al modo como el tribunal alemán ha argumentado su cambio jurisprudencial y centrándonos ya de nuevo en Derecho al olvido II, es de

terminado" por el derecho de la Unión. No es, en efecto, precisamente transparente lo que signifique regular de manera completa y acabada una determinada materia (Wendel, 2020: 1406-1408). Sabemos, ciertamente, que la decisión marco relativa a la orden de detención y entrega regula acabadamente la materia en cuestión, porque así consta en Melloni. La pareja formada por las dos sentencias de 6 de noviembre de 2019 pretende ilustrar este binomio. Para ello contrapone, de un lado, un supuesto en el que el derecho de la Unión (la Directiva 95/46, de protección de datos) deja por excepción un espacio abierto a la regulación del Estado miembro (Derecho al olvido I). Y de otro lado un supuesto en el que la referida normativa europea no contiene tal cláusula de apertura (Derecho al olvido II). En concreto el art. 9 de la Directiva disponía que: «En lo referente al tratamiento de datos personales con fines exclusivamente periodísticos o de expresión artística o literaria, los Estados miembros establecerán, respecto de las disposiciones del presente capítulo, del capítulo IV y del capítulo VI, exenciones y excepciones sólo en la medida en que resulten necesarias para conciliar el derecho a la intimidad con las normas que rigen la libertad de expresión». Ahora bien, si una disposición de este tipo permite alcanzar autónomamente la conclusión del tribunal alemán es algo que no deja de ser opinable a partir del conjunto de la jurisprudencia del TJUE sobre la materia.

39 Es una situación expresivamente reflejada en el título del comentario de Wendel (2020: 1383): «The two-faced Guardian». Téngase en cuenta que aquí se ha dejado de lado, en tanto que menos interesante para el observador externo, el modo de argumentar de las Salas, tanto la una como después la otra, respecto de su decisión de no elevar el cambio jurisprudencial al Pleno.

Cit. nota 5 . 
comenzar subrayando que, a diferencia del tribunal vienés, Karlsruhe no se preocupa por argumentar la razón de que el cambio jurisprudencial tenga lugar en este momento. La realidad es que insistir en la llegada de la Carta tiene poco sentido cuando lleva ya de diez años de vigencia. Una reflexión sobre las consecuencias del cambio de paradigma se encuentra aquí completamente ausente. $Y$ es que el argumento fuerte del tribunal alemán es de otro mundo, como se verá a continuación.

La Sala Primera del Tribunal Constitucional Federal, al igual que un año más tarde su Sala Segunda, declararán que una dimensión esencial de su propia razón de ser estriba en la garantía de los derechos fundamentales. Ahora bien, estos «derechos fundamentales» no son tales porque formen parte de la Grundgesetz de la República Federal de Alemania: son los derechos fundamentales, si se puede hablar así, como tales, es decir, con independencia de que los mismos encuentren su asiento, positivizados, en uno u otro ordenamiento jurídico, en nuestro caso, en el alemán o en el de la Unión. La garantía de estos derechos fundamentales, en su abstracción, forma parte esencial de la identidad de la jurisdicción constitucional alemana. Eso lleva al tribunal a declarar que los «derechos fundamentales» sin más precisión no puedan ser adecuadamente protegidos en el ámbito de soberanía alemán si él mismo no extiende su tutela cuando se sitúan en otro ordenamiento, en concreto en el de la Unión ${ }^{41}$. Y es que, en alguna medida, es secundario donde los derechos fundamentales encuentren plasmación escrita. Sin que se diga expresamente, no debe olvidarse la realidad subyacente de los límites absolutos al poder

41 Valgan los siguientes pasajes: «La garantía de una efectiva protección de los derechos fundamentales forma parte de las tareas centrales del Tribunal Constitucional Federal [...] (punto 58). La Verfassungsbeschwerde se halla concebida conscientemente de forma amplia y comprensiva [...] (punto 60) [...] una protección constitucional de los derechos fundamentales sólo se garantiza si el Tribunal Constitucional Federal incorpora a su canon de control los derechos fundamentales de la Unión con ocasión del control de la aplicación del derecho por los tribunales ordinarios [...] (punto 63) [...] Dado que en la actualidad la protección constitucional de los derechos fundamentales en parte se encuentra rebasada como consecuencia de la primacía de aplicación del Derecho de la Unión, no hay motivo alguno para negarle a las ciudadanas y ciudadanos este remedio procesal [...] (punto 66) en tanto que garante de una completa protección de los derechos fundamentales en el interior del Estado, el Tribunal Constitucional Federal ha de controlar a los tribunales ordinarios a este respecto». En todos ellos se hace alusión a los derechos fundamentales como una categoría abstracta, como pueda ser la democracia o el Estado de derecho, en definitiva, como una categoría desligada de un ordenamiento jurídico preciso, en el que, como tales derechos fundamentales, encuentra su conexión de sentido. 
de reforma constitucional (art. 79.3 GG): el carácter suprapositivo de los derechos fundamentales está en el ADN de la Grundgesetz.

Esta comprensión de la categoría derechos fundamentales se completa con otro argumento de factura no menos alemana, la llamada «responsabilidad de integración» (Integrationsverantwortung), una categoría también marca de la casa ${ }^{42}$ y que, en contra de lo que el término parece dar a entender, apuntaba hasta ahora más bien a la garantía de los límites de la integración europea (ex art. 23 GG) que no a la de la integración misma (Wendel, 2020: 1422-1423). Ocurre, sin embargo, que la «responsabilidad de integración» pasa ahora a tener un entendimiento positivo, en el sentido de fundamentar la garantía de la CDF (Hofmann y Heger, 2021: 5-6).

El postulado del equivalente funcional sirve también para apoyar la jurisprudencia ${ }^{43}$. La CDF cumple una función equivalente al título I GG. Se argumenta de manera diferente a como lo hacía el Verfassungsgerichtshof, pero el resultado es muy parecido: de algún modo los derechos de una y otra serie son intercambiables, produciéndose un efecto de reducción implícita del alcance de la extensión del amparo. Posiblemente esto explique que el tribunal alemán no se haya detenido en considerar expresamente qué contenidos sustantivos de la CDF son aptos para la Verfassungsbeschwerde alemana.

Por lo que hace ya a la construcción de la nueva competencia, si algo la caracteriza frente a la austriaca es una primera advertencia: nada de esto va a implicar que Karlsruhe vaya a debilitar su responsabilidad en los llamados control de identidad y control ultra vires de los actos de la Unión ${ }^{44}$. La garantía de los derechos de la Carta no será absolutamente incondicional. Operará solamente en la medida en que supere dichos controles, debiendo hacerse notar que esto no tiene el carácter de una simple advertencia ritual. De hecho, Euroorden III argumenta muy extensamente (puntos 57-69) por qué la protección ofrecida por el art. $4 \mathrm{CDF}$ (prohibición de tratos inhumanos o degradantes) no es inferior a la exigida por la Grundgesetz en términos de identidad constitucional alemana. Solo así puede prosperar una petición de amparo respondida con la herramienta de la Carta.

Dicho esto, el elemento más característico de la construcción alemana es su pretensión de dejar fuera la extensión del amparo (en todo caso por el momento) a los procesos de control de normas. El tribunal insiste en que pretende exclusivamente llevar a cabo un control sobre la interpretación

42 Sentencia Lisboa de la Sala Segunda, de 30 de junio de 2009, BVerfGE 123, 267, punto 245 .

43 Punto 59.

44 Punto 49. 
efectuada por la jurisdicción que aquíllamamos ordinaria (Fachgerichtsbarkeit) de los derechos de la Carta ${ }^{45}$. Dicho de otra manera, se trata de una garantía ceñida al amparo frente a resoluciones judiciales (Urteilsgerichtsbarkeit), tan universal este como el amparo español ${ }^{46}$.

Desde luego, esta asunción de competencia jurisdiccional va acompañada igual que en el caso austriaco de la disponibilidad del tribunal germano en orden a elevar cuestiones prejudiciales relativas a los derechos de la Carta en idénticos términos a lo que es el caso de la jurisdicción ordinaria. Cosa distinta es su efectiva disponibilidad a hacerlo. En esto también se asemeja al tribunal austriaco. Si la circunstancia de que se trate de un acto aclarado está exhaustivamente argumentada en Euroorden III, no tan convincente resulta en Derecho al Olvido II. Por lo demás, al igual que la sentencia austriaca, las sentencias alemanas dejan incólume el amplio derecho de los tribunales nacionales, o el deber en los casos de última instancia, de elevar cuestiones prejudiciales al TJUE: Melki y Abdeli es debidamente citado, sin que conste que la jurisdicción ordinaria, a diferencia de lo ocurrido en Austria, haya trasladado una eventual consulta al TJUE.

Por fin, preciso es señalar cómo dentro del argumentario de la sentencia tampoco ocupa un lugar destacado la referencia a las lagunas mostradas por el derecho de la Unión en punto a tutela judicial y, en definitiva, a vías de recurso disponibles en reivindicación de derechos fundamentales. Derecho al olvido II se hace un eco más que modesto ${ }^{47}$.

45 Decididamente escéptico en este punto, Schneider (2020: 21-24).

46 Con el importante matiz de la categoría, de contorno problemático, del llamado «Derecho constitucional específico" (spezifisches Verfassungsrecht) como específico ámbito de control, valga la redundancia, del juez constitucional, categoría con la que se pretende no interferir en los ámbitos propios de cada orden jurisdiccional aún regidos por los mandatos constitucionales. Derecho al olvido II (puntos 124-136) es un excelente ejemplo de cómo el tribunal opera con esta categoría en el control de una operación de ponderación entre derechos fundamentales llevada a cabo por el juez ordinario, en este caso dándola por suficiente desde la perspectiva del control que a él le corresponde. Una excelente presentación de la espinosa categoría ante un público especializado, por más que físicamente distante, en Grimm, 2014: 339-376.

47 Punto 61: «La señalada laguna en la protección de los derechos relativamente a la jurisdicción ordinaria no se cubre por medio de vías de recurso correspondientes a nivel de Derecho de la Unión. Los particulares carecen de la oportunidad de denunciar de manera directa ante el TJUE una vulneración de los derechos fundamentales de la Unión por parte de la jurisdicción ordinaria de los Estados miembros» (traducción nuestra). Eso es todo. 
La doctrina alemana ha sido en su conjunto más solidaria con el cambio jurisprudencial por comparación con su precedente austriaco. La crítica, y no generalizada, se ha situado en el modo de introducir esta extensión de la Verfassungsbeschwerde a la CDF, es decir, el que haya sido Karlsrube y no el legislador (posiblemente mediante reforma constitucional) quien la haya llevado a cabo ${ }^{48}$. Pero el argumentario y la construcción han sido esencialmente aceptados.

\section{LA PULSIÓN QUIETISTA: MADRID, 2021}

Por contraste con los pasos dados por los dos tribunales constitucionales hasta ahora considerados, el español ha permanecido hasta ahora quieto. Hablar de pulsiones quietistas puede parecer contradictorio. En ocasiones, sin embargo, no hacer nada cuando tanto movimiento hay alrededor puede requerir voluntad y esfuerzo para precisamente mantenerse en el mismo sitio. Tal parece la conducta de un Tribunal Constitucional nacional fundamentalmente reacio a adentrarse por cualquier senda que conduzca a precisar del auxilio del Tribunal de la Unión en la interpretación del derecho en su caso llamado a aplicar, y en último término a ofrecer sus servicios a la CDF. Ocurre, sin embargo, que el tribunal español parte de buenos argumentos para mantenerse fiel a su doctrina. En realidad, son los mismos que fundamentaban la jurisprudencia, previa al cambio, de los tribunales hasta ahora considerados. Esto no debiera ser obstáculo para abrir un debate al respecto a la vista de la evolución producida en la jurisprudencia de los tribunales vecinos. Comenzando, sin embargo, por el principio:

El Tribunal Constitucional español, desde sus inicios, nunca ha dejado de destacar su condición e incluso su identidad como tribunal constitucional, es decir, de tribunal singular que encuentra su razón de ser en la Constitución y precisamente en la Constitución española de 29 de diciembre de 1978, antes, pues, que cualquier idea abstracta de constitución. Esta Constitución es la aludida singularmente en el art. 9.1 CE al proclamar el sometimiento a la misma de los ciudadanos y de los poderes públicos. Ella es igualmente la aludida cuando su Ley Orgánica (art. 1.1) se refiere el Tribunal Constitucional, único en su orden, como intérprete supremo de la Constitución e implícitamente, supremo garante de su normatividad. Y esto es lo que late en la proclamación de que dicho tribunal está exclusivamente sometido a la Constitución y a su Ley Orgánica. De forma aún más particular le corres-

48 Referencias a las voces críticas en Hofmann y Heger (2021: nota 119). 
ponde la tutela de los derechos fundamentales que su título I reconoce, en especial mediante el remedio judicial del recurso de amparo constitucional como materia objeto de regulación en la Ley Orgánica justo tras el control de normas.

A partir de aquí será la jurisprudencia constitucional quien subraye el carácter exclusivo de su competencia por contraste con otros posibles cánones de control: la Constitución de 1978 como su alfa y omega ${ }^{49}$. La interpretación y garantía de otros ordenamientos no es de su incumbencia ${ }^{50}$. Esa responsabilidad es ya del Poder Judicial ordinario del Estado (título VI CE), vale decir, de los jueces y tribunales, quienes normalmente lo hacen, no tanto en una función autónoma de control de validez, sino en el marco de un litigio y en el curso del proceso ordinario de selección de la norma aplicable. Descartar o desplazar la aplicación de una norma como efecto del principio de primacía, en un contexto que no tiene que ser de estricta jerarquía entre categorías de normas, está ciertamente lejos del resultado de declaración de invalidez de una norma, privilegio, como regla, del Tribunal Constitucional. Con todo, la garantía de esta primacía, de la que la misma Constitución, salvo supuestos "difícilmente concebibles»" ${ }^{51}$, no se libra, queda fuera del control del juez constitucional.

Es así como el Tribunal Constitucional difícilmente se verá en la tesitura de estar aplicando otro Derecho que la Constitución, o su Ley Orgánica, en el curso de un proceso constitucional. Lo cual es tanto como decir, más en concreto, que el juez constitucional español escapa por principio a la condición de órgano jurisdiccional en el sentido del art. 267 TFUE, vale decir, y por definición, órgano de última instancia llamado a estar obligado a formular una cuestión prejudicial al TJUE.

Todo esto requiere una primera matización. El tribunal español, como antes que él el alemán, ha considerado un supuesto de vulneración del derecho a la tutela judicial la no elevación de una cuestión prejudicial al TJUE para

49 Con el sabido desafortunado calificativo: «[...] la eventual infracción de la legislación comunitaria europea no convierte en litigio constitucional lo que sólo es un conflicto de normas infraconstitucionales que ha de resolverse en el ámbito de la jurisdicción ordinaria» (STC 28/91, FJ 5). Y por lo que hace al amparo: «[...] es patente que los motivos de amparo han de consistir siempre en lesiones de los derechos fundamentales y libertades públicas enunciadas en los arts. 14 a 30 C.E.» (STC 64/91, FJ 4). Una completa recopilación de la doctrina del Tribunal Constitucional relevante en la materia, presentada por el propio tribunal: Prontuario de jurisprudencia del Tribunal Constitucional sobre el derecho de la Unión Europea, accesible en https://bit. ly/3nWmMtg.

51 DTC 1/2004, FJ 4. 
parte de la jurisdicción ordinaria en términos que suponen desatender la doctrina CILFIT de dicho tribunal ${ }^{52}$. De hecho, así lo ha apreciado, respecto del Tribunal Supremo, en el asunto que dio lugar a su todavía reciente Sentencia $37 / 2019^{53} y$, como consecuencia última de esta, a la pendiente sentencia de Luxemburgo en la cuestión prejudicial elevada por dicho Tribunal Supremo. La matización se impone desde el momento en que amparar frente a la conducta omisiva del juez nacional exigirá una interpretación del derecho de la Unión ${ }^{54}$. Las consideraciones del Abogado General Bobek en sus conclusiones, ya pronunciadas en la referida cuestión prejudicial, hablan por sí mismas ${ }^{55}$.

En contraste con el supuesto anterior, el afamado caso Melloni, citado desde el principio, es distinto: la hasta ahora única cuestión prejudicial formulada por el Tribunal Constitucional tiene otro origen. Por recordarlo brevemente, en línea de principio la decisión marco regulando la orden de detención y entrega, en su modificación de 2009, debía imponerse a la doctrina constitucional

52 Prontuario, 13-14: «A) El DUE no es canon de constitucionalidad autónomo».

53 Véase Prontuario, pp. 17-23: «Decisión de la jurisdicción ordinaria sobre el planteamiento de cuestión prejudicial».

54 Como parece resultar con naturalidad de la lectura del siguiente pasaje de la STC 37/2019, FJ 6: «Por ello, en el procedimiento a quo no concurrían los presupuestos necesarios para apreciar que la doctrina emanada en las referidas sentencias del Tribunal de Justicia de la Unión Europea constituía un "acto aclarado" respecto del problema interpretativo suscitado y, por tanto, el órgano judicial no estaba dispensado de plantear cuestión prejudicial ante el mencionado Tribunal de Justicia».

55 Asunto Viesgo Infraestructuras Energéticas, SA (C-683/19), conclusiones de 15 de abril de 2021 (ECLI:EU:C:2021:300), punto 40: «[...] si el tribunal superior o el tribunal constitucional nacional ante el cual se ha interpuesto un recurso extraordinario comienza a revisar si el tribunal de última instancia ha aplicado correctamente la excepción del "acto claro" de la jurisprudencia CILFIT, lo más probable es que ese propio tribunal de recurso haya de interpretar el Derecho de la Unión. (27) Sin embargo, en ese momento se convierte en "órgano jurisdiccional' a efectos del artículo 267 TFUE. Además, dado que dicho tribunal de recurso, por definición, ha de ser un órgano jurisdiccional en el sentido del artículo 267 TFUE, párrafo tercero, (28) este asumirá todas las obligaciones y responsabilidades que se derivan de su condición, incluida, en su caso, la obligación de remitir por sí mismo una petición de decisión prejudicial». Merece reproducir también la nota 27 de las propias conclusiones: «[...] Por otro lado, en la comprobación específica de si existía o no un "acto claro" ante un tribunal de última instancia en un caso concreto, no puedo dejar de admirar la capacidad para revisar el fondo de un asunto sin entrar en él e interpretarlo. Es como el sueño de Schrödinger hecho por fin realidad: tener la habilidad de decir con certeza si el gato (de CILFIT) está vivo o muerto sin siquiera tener que abrir la caja (del Derecho de la Unión)». 
española relativa a las condenas penales en ausencia. Esto suponía que el juez constitucional nacional debía dejar sin efecto la doctrina contenida en la STC 91/2000, que había llegado a calificar la proscripción de este modo de condenas penales como parte del «contenido absoluto» del art. 24.2 CE. Para ponérselo más difícil al TJUE, el tribunal pedía contrastar la reforma de 2009 con la garantía del «superior nivel de protección» del ordenamiento nacional, preservado en el art. 53 CDF. La triple negación de la sentencia recaída en Melloni, pero muy en particular la tercera negativa, convirtió el asunto Melloni en el trauma Melloni ${ }^{56}$. De sobra es sabido: el superior nivel de protección no se impone en supuestos de regulación exhaustiva de la materia por el legislador de la Unión.

En estas condiciones, el tribunal redujo la respuesta recibida a poco más que un valioso auxilio interpretativo, siempre con el trasfondo del art. 10.2 $\mathrm{CE}^{57}$. Una cosa puede decirse en conclusión, y es que tras su experiencia con Melloni cabe imaginar un punto de desafección por parte del juez constitucional español respecto de los derechos de la Carta. En todo caso, una predisposición por su parte a facilitar eventuales recortes no parece por sí misma evidente.

La conclusión nítida de todo esto es que, con arreglo a su doctrina constante y pacífica, el Tribunal Constitucional español inadmitirá inmediatamente a trámite cualquier demanda de amparo basado en otra cosa que no sean los derechos y libertades reconocidos en la Constitución española y específicamente los delimitados en el art. 53.2 CE. A partir de aquí se plantea la cuestión anunciada desde el principio, es decir, la de si es previsible o en

56 A notar el interminable y atormentado FJ 4 de la STC 132/2000, de 23 de diciembre, en recurso de amparo elevado al Pleno. Cfr. De la Quadra-Salcedo Janini y Rodríguez Fernández, 2020: 99-104. Recuérdese todavía cómo la Declaración del Tribunal Constitucional relativa al Tratado por el que se establece una constitución para Europa (FJ 6) había declarado con toda naturalidad: "[...] claramente se advierte que la Carta se concibe, en todo caso, como una garantía de mínimos, sobre los cuales puede desarrollarse el contenido de cada derecho y libertad hasta alcanzar la densidad de contenido asegurada en cada caso por el Derecho interno» (DTC 1/2004, de 13 de diciembre, FJ 4).

57 Las frases del Prontuario son expresivas: «De esta forma el TC se vio a obligado a dilucidar de qué manera debía integrar la jurisprudencia del TJUE [...] El TC, en atención al valor hermenéutico del art. 10.2 optó por asimilar la jurisprudencia del TJUE [...]» (10); «[...] existen supuestos en los que para desempeñar correctamente su función como órgano de garantías constitucionales el TC, en tanto que tiene la consideración de órgano jurisdiccional a los efectos del art. 267 TFUE, puede considerar conveniente elevar una cuestión prejudicial al TJUE. Así se decidió [...]» (10) (cursivas nuestras). 
todo caso si es conveniente que el tribunal español siga la senda marcada en Viena y Karlsruhe.

La cuestión resultará desde el principio más transparente si se formula en términos prácticos. Tal sería el supuesto de que el tribunal español se viera en la tesitura del tribunal alemán en el asunto Euroorden III. ¿Qué ocurriría si un juez español accediera a una solicitud de entrega proveniente de un Estado miembro cuyos centros de detención posiblemente no reúnen las mínimas condiciones exigibles derivadas del art. $4 \mathrm{CDF}$ ? En el estado ya conocido de la jurisprudencia del TJUE ${ }^{58}$ no es previsible que esto suceda, en todo caso no es previsible que suceda sin un previo planteamiento de una cuestión prejudicial por parte del juez español. Ahora bien, si a pesar de todo estas circunstancias se dieran, el Tribunal Constitucional tendría poco que hacer como consecuencia de la jurisprudencia combinada del TJUE y la suya propia: regirían los derechos fundamentales de la Carta, quedando desplazados los de la Constitución, parámetro exclusivo rebus sic stantibus de la jurisdicción nacional de amparo. En estas condiciones, el Tribunal Constitucional no podría ir más allá de declarar vulnerado el derecho a la tutela judicial por falta de planteamiento de la cuestión prejudicial por parte del juez de entrega. A menos que se hubiera optado directamente por la demanda individual ante el TEDH con invocación de la clara jurisprudencia de este últimos?.

Ciertamente, el Tribunal Constitucional puede sentirse cómodo con este diseño, sin que haya en este momento motivos para pensar lo contrario. Pero igual considera que el cuadro de conjunto no es satisfactorio, por ejemplo, desde la perspectiva de dilaciones indebidas en materia de garantía de derechos fundamentales. O que conviene evitar condenas innecesarias del Reino de España. O sencillamente porque los derechos de la Carta merecen ser mejor protegidos ad intra. Pues los supuestos pueden multiplicarse en el estado de desarrollo del derecho de la Unión. ¿Llegaría su insatisfacción al punto de imitar a sus colegas centroeuropeos?

No me lo parece, realmente, sin que falten síntomas para apoyar esta impresión. La identidad de tribunal constitucional, de tribunal «para la Constitución", es muy fuerte en su caso, con buenos asideros en la propia Constitución y en la ley orgánica. Por otra parte, y en términos funcionales, se trata de un tribunal demasiado escorado, por necesidad, a la jurisdicción de conflictos terri-

58 Dimitru-TudorDorobantu, Sentencia de 15 deoctubrede2019(C-128/18,ECLI:EU:C: 2019:857); ML y Generalanwalschaft Bremen (C-228/18 PPU, ECLI:EU:C: 2018:589).

59 La misma jurisprudencia del TEDH que cita Euroorden III: Muršić contra Croacia, Sentencia de 20 de octubre de 2016. 
toriales, al menos en términos comparativos. De otro lado, el tribunal ha vivido desde casi el primer día la gestión del amparo constitucional con el sentimiento de verse desbordado por su cantidad. Ha potenciado en consecuencia el factor calidad, lo que se llamó la objetivación del amparo, hasta llegar a la temible aduana que constituye hoy la demostración de la trascendencia constitucional de la demanda (art. 49.1 LOTC). Y, si bien se mira al fundamento jurídico 6 de la STC140/2018, de 20 de diciembre, casi se diría que, con la genericidad de su lenguaje, está animando a la jurisdicción ordinaria a gestionar directamente la protección de los derechos fundamentales y libertades públicas con el mantra de la mera selección del derecho aplicable al caso ${ }^{60}$.

En suma, no parece previsible que el tribunal español esté próximo a moverse de su centrismo constitucional. Y la realidad es que, en todo caso, no hace mal en abstenerse de introducir un cambio jurisprudencial de la enjundia que se ha podido constatar en las latitudes examinadas. Hemos visto los argumentos de uno y otro tribunal centroeuropeo, inspirados por una u otra pulsión nacional. Y la verdad es que no son hasta tal punto inobjetables que merezcan un trasplante automático a nuestras latitudes. No es necesario extenderse mucho: con total independencia de lo que la jurisprudencia comentada supone en términos de avance en la garantía jurisdiccional de la CDF, no cabe negar que, por lo que hace a los argumentos sobre los que está construida, hay algunos elementos de arbitrismo, en un caso, y otro de excesivo principialismo, en el otro. Pero más allá de la dificultad de asumir cada uno de los referidos argumentos, está la dificultad de que una reformulación del amparo de este calibre deba llevarla a cabo este Tribunal Constitucional, y ni aun otro, si se me apura. En mi opinión este es un caso de reserva de ley, al menos.

En este sentido, la ordenación del ámbito de la jurisdicción constitucional española tiene interés a los efectos que ahora importan. Más allá de las protestas de exclusividad, la realidad es que la propia Constitución relativizó desde el principio esta categorización al abrir el ámbito de la competencia del tribunal, de modo genérico, a materias fijadas por Ley Orgánica (art. 161.1.d CE). Y no se puede decir que este legislador orgánico se haya abstenido de hacer uso de esta facultad, ampliando la jurisdicción del Tribunal Constitucional. Baste remitir a añadidos tales como el monopolio de control de las normas forales ${ }^{61}$ o la garantía de la autonomía local ${ }^{62}$. Todo ello dejando de lado la encomienda de tareas procesales enojosas, por así decir ${ }^{63}$.

\footnotetext{
60 Alonso García (2020: 13-51).

61 Disposición adicional 5a LOTC.

62 De los conflictos en defensa de la autonomía local: arts. 75 bis a 75 quinque LOTC.

63 Art. 92 LOTC.
} 
Con estos precedentes, entiendo que el legislador orgánico podría sin dificultad incorporar parte del contenido sustantivo de la CDF a la tutela por vía de amparo ${ }^{64}$, posiblemente sin necesidad de introducir cambios en los procedimientos de declaración de inconstitucionalidad.

Eso no quiere decir que el legislador no tenga que argumentar este cambio, se entiende en términos de racionalidad del cambio legal. Se trataría con este cambio legislativo de cubrir la actual laguna en el sistema de remedios judiciales a disposición de los particulares a la hora de reivindicar los derechos fundamentales que la CDF reconoce en el espacio constitucional europeo. No lo haría desde luego con efectos en toda la Unión, pero sí reforzaría considerablemente la iniciativa de los colegas centroeuropeos. Ciertamente no tendría por sí consecuencias para las vías de recurso frente a la otra vertiente aplicativa de la CDF, a los actos de los órganos e instituciones de la Unión: Pero pondría al legislador de la Unión frente a un espejo cuando menos incómodo.

\section{CONCLUSIONES}

Una especial preocupación por mejorar las vías de recurso a disposición de los ciudadanos europeos en orden a hacer valer sus derechos fundamentales derivados de la Carta es algo que cuesta descubrir tanto en el cambio jurisprudencial producido hace nueve años en Viena como en el producido hace dos en Karlsruhe. Por el contrario, tanto en la actuación preventiva del tribunal austriaco como en la reactiva del alemán ${ }^{65}$ (scil. Akerberg), lo que ante todo se advierte es el propósito de preservar su respectiva posición en la garantía de los derechos fundamentales. Los derechos de la Carta son así objeto de repatriación por lo que a su interpretación y garantía se refiere, sin que las protestas de disposición a asumir la condición de órgano jurisdiccional afectado por el art. 267.4 TFUE tengan la fuerza de convicción deseable. Es así como tampoco se advierte que desde Luxemburgo se haya saludado con particular entusiasmo esta oferta de colaboración de los tribunales constitucionales nacionales en forma de puesta a disposición de las vías de recurso nacionales destinadas a una reforzada garantía de los derechos fundamentales internos. No es aventurado pensar que los tribunales nacionales en cuestión

64 Y, de paso, los derechos y libertades de la Sección Segunda del Capítulo II del Título I de nuestra Constitución: eso permitiría eliminar una anomalía por contraste con los tribunales vecinos.

65 Reacción bien temprana, casi «a vuelta de correo», debidamente analizada en Arzoz (2016). Aplicado a Derecho al olvido II: «an instance or resistance» (Burhardt, 2020: 8). 
van a abstenerse de entrar a conocer de amparos en los supuestos en los que exista un claro riesgo de verse obligados a plantear una cuestión prejudicial. En esta tesitura, y si esta nueva competencia de los tribunales constitucionales sigue adelante, cabe contar con un «tira y afloja» entre los unos y el otro ${ }^{66}$.

Con independencia de la consideración anterior, es un hecho que los ciudadanos europeos de cualquier origen nacional disponen ahora de la extraordinaria vía de recurso que supone la Verfassungsbeschwerde, cada vez que necesiten reivindicar los derechos y libertades consagrados en la CDFUE frente a los actos de poder público emanados, de momento, en dos Estados miembros en aplicación del derecho de la Unión y en supuestos en los que, de otro modo, no hubieran podido invocar los derechos fundamentales reconocidos en las respectivas constituciones. Por lo que hace a estos Estados, la normatividad de la Carta deja de estar como hasta ahora esencialmente dependiente de la sensibilidad de los órganos jurisdiccionales nacionales por la vía de la cuestión prejudicial para encontrar un nuevo puerto de refugio en el esencialmente democrático remedio judicial que la demanda individual, sin entrar ahora en cargas de otro tipo, por principio supone.

Dicho esto, es de notar que, con el estado de cosas actual se genera una llamativa, por no decir problemática, asimetría territorial en la garantía de los contenidos sustantivos de la Carta en razón de los poderes públicos implicados, con la consiguiente repercusión inmediata en un elemento básico del status civitatis europeo. En otras palabras, desde la perspectiva del espacio constitucional europeo se plantea la cuestión de cuánta asimetría territorial resulta soportable en una materia tan sensible como la que nos ocupa.

Es sobre todo aquí donde conviene situar el debate en torno a lo que se ha calificado como la pulsión quietista madrileña. Los recientes cambios efectuados por vía jurisprudencial en otros Estados miembros en el ámbito específico de la jurisdicción de amparo sitúan a la jurisdicción de amparo española ante la tesitura, no fácilmente evitable, de operar un ejercicio de introspección, una operación en la que no debiera encontrarse sola. La cuestión es lo suficientemente técnica como para encontrar el oído del legislador. En fin, más allá del análisis en términos de costes y beneficios, un enfoque que merezca ser calificado de europeo no podrá perder la perspectiva de su repercusión sobre la calidad transversal de los derechos fundamentales en la Unión.

66 Después de todo, Francia ya cuenta con una condena por incumplimiento basado en la falta de planteamiento de una cuestión prejudicial por parte del Consejo de Estado, Sentencia de 4 de octubre de 2018 (Comisión contra República Francesa, C-416/17, ECLI:EU:C:2018:811), punto segundo del fallo. 


\section{Bibliografía}

Ahumada Ruiz, M. (2020). La jurisdicción constitucional y las jurisdicciones europeas. En busca del equilibrio. En Asociación de Letrados del Tribunal Constitucional (coord.). Cuatro décadas de jurisprudencia constitucional: Los retos (pp. 247-274). Madrid: Centro de Estudios Políticos y Constitucionales.

Alonso, R. (2020). El control de convencionalidad: Cinco interrogantes. Revista Española de Derecho Constitucional, 119, 13-51. Disponible en: https://doi. org/10.18042/cepc/redc.119.01.

Arzoz, X. (2015). La tutela de los derechos fundamentales de la Unión Europea por el Tribunal Constitucional. Madrid: Instituto Nacional de Administración Pública.

- (2016). Karlsruhe rechaza la Doctrina Melloni del Tribunal de Justicia y advierte con el control de la identidad constitucional (comentario a la Sentencia del Tribunal Constitucional Federal Alemán de 15 de diciembre de 2015, 2 BvR 2735/14). Revista Española de Derecho Europeo, 58, 109-141.

Burchardt, D. (2020). Backlash against the Court of Justice of the EU? The Recent Jurisprudence of the German Constitutional Court on EU Fundamental Rights as a Standard of Review. German Law Journal, 21, 1-18. Disponible en: https://doi.org/10.1017/glj.2020.16.

Franzius, C. (2015). Strategien der Grundrechtsoptimierung in Europa. Europäische Grundrechte Zeitschrift, 42 (5), 139-153.

Grabenwarter, Chr. (2016).Verfassungsgerichtsbarkeit in Europa: Institutionen. En A. v. Bogdandy, Chr. Grabenwarter, P. M. Huber (dirs.). Handbuch Ius Publicum Europaeum. VI (pp. 413-469). Heidelberg: C.F. Muller.

Grimm, D. (2014). Die Urteilsverfassungsbeschwerde und das Verhältnis von Verfassungsgericht und Fachgerichten in Deutschland. Seoul Law Journal, 55, 339376.

Hofmann, R. y Heger, A. (2021). Zur neuen Grundrechte-Architektur im europäischen Mehrebenensystem. Europäische Grundrechte Zeitschrift, 48, 1-11.

Iglesias, S. (2012). The Court and the Charter: The Impact of the Entry into Force of the Lisbon Treaty on the ECJ's Approach to Fundamental Rights. Common Market Law Review, 49, 1565-1612.

Kottmann, M. (2010). Plaumanns Ende: Ein Vorschlag zu Art. 263.4 AEUV. Zeitschrift für ausländisches öffentliches Recht und Völkerrecht, 70, 547-566.

Orator, A. (2015). The decision of the Austrian Verfassungsgerichtshof on the EU Charter of Fundamental Rights: An Instrument of Leverage or Rearguard Action? German Law Journal, 16 (6), 1429-1447. Disponible en: https://doi. org/10.1017/S2071832200021209.

Pöschl, M. (2012). Verfassungsgerichtsbarkeit nach Lissabon. Anmerkungen zum Charta-Erkennnis des VfGH. Zeitschrift für öffentliches Recht, 67, 587-609. Disponible en: https://doi.org/10.1007/s00708-012-0156-0. 
Prechal, S. (2011). Redefining the Relationship between, Rewe-effectiveness' and Effective Judicial Protection. Review of European Administrative Law, 4, 31-50. Disponible en: https://doi.org/10.7590/REAL_2011_02_03.

Quadra-Salcedo, T. y Rodríguez, I. (2020). La relevancia de la doctrina de las vulneraciones indirectas de los derechos fundamentales. ¿Es asumible una pluralidad de contenidos de la dignidad humana? Revista Española de Derecho Europeo, 76, 73-107. Disponible en: https://doi.org/10.37417/REDE/num76_2020_528.

Rauchegger, C. (2020). National Constitutional Courts as Guardians of the Charter: A Comparative Appraisal of the German Constitutional Courts's Right to be Forgotten Judgments. Cambridge Yearbook of European Legal Studies, 22, 258278.

Sarmiento, D. (2010). Cilfit and Foto-Frost: Constructing and Deconstructing Judicial Authority in Europe. En M. Poiares y L. Azoulai (eds.). The Past and Future of EU Law, (pp. 192-200). Oxford: Bloomsbury Publishing.

Schneider, K. (2020). The Constitutional Status of Karlsruhe's Novel «Jurisdiction» in EU Fundamental Rights Matters: Self-inflicted Institutional Vulnerabilities. German Law Journal, 21, 19-26. Disponible en: https://doi.org/10.1017/ glj.2020.17.

Tribunal Constitucional de España, Prontuario de jurisprudencia del Tribunal Constitucional sobre el Derecho de la Unión Europea. Disponible en: https://bit. ly/3b9acld.

Visser, M. (2015). Juggling centralized constitutional review and EU primacy in the domestic enforcement of the Charter: A. v. B. Common Market Law Review, 52, 1309-1338.

Wendel, M. (2020). The two-faced guardian - or how one half of the German Federal Constitutional Court became a European Fundamental Rights Court. Common Market Law Review, 57, 1383-1426. 